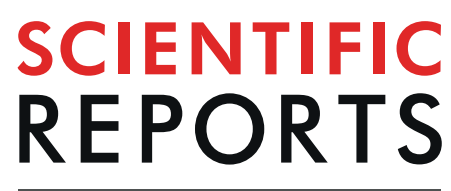

natureresearch

Check for updates

\title{
Effect of life stage and pesticide exposure on the gut microbiota of Aedes albopictus and Culex pipiens $\mathrm{L}$
}

Elijah O. Juma ${ }^{1 凶}$, Brian F. Allan ${ }^{1}$, Chang-Hyun Kim², Christopher Stone ${ }^{2}$, Christopher Dunlap ${ }^{3}$ \& Ephantus J. Muturi ${ }^{3}$

Pesticides commonly contaminate the aquatic environments inhabited by mosquito juveniles. However, their role in shaping the mosquito microbiota is not well understood. We hypothesized that environmentally relevant concentrations of atrazine, permethrin and malathion will mediate a shift in the mosquito gut bacterial community structure due to their toxic effect on the aquatic bacterial communities, and reduce mosquito gut bacterial diversity by enriching pesticide-degrading bacterial communities over susceptible taxa. Illumina MiSeq sequencing of the V3-V4 hypervariable regions of the $16 \mathrm{~S}$ rRNA gene was used to characterize the microbial communities of larval and adult stages of the two mosquito species and the water samples from microcosms treated with each of the pesticides, separately. Bacterial community composition differed by sample type (larval stage vs. adult stage) and water sampling date (day 3 vs. day 7), but not by pesticide treatment. In larval stages, bacterial OTU richness was highest in samples exposed to malathion, intermediate in permethrin, and lowest in controls. Bacterial richness was significantly higher in larval stages compared to adult stages for all treatments. This study provides a primer for future studies evaluating mosquito microbial responses to exposures to chemical pesticides and the possible implications for mosquito ecology.

One of the central goals of ecology is to understand patterns of species abundance and diversity in communities and ecosystems, and how these patterns are impacted by anthropogenic alterations ${ }^{1-3}$. Pesticides are a common source of environmental disruption, and thousands of different pesticides are used around the world to improve crop production and human health. These pesticides often enter the aquatic habitats, including those that are not intended or legally registered for application to aquatic systems. Consequently, there is considerable research effort to understand and predict the impacts of anthropogenic chemical pesticides on non-target aquatic organisms $^{4-7}$.

Atrazine, permethrin, and malathion are three of the most frequently used pesticides, and their residues are commonly detected in groundwaters of the United States ${ }^{4,8,9}$. Although malathion is still used in agricultural pest control, it is no longer used in vector control in the United States. These three pesticides span a broad range of mode of action and uses. Atrazine, a triazine herbicide, is used globally to control broadleaf weeds by inhibiting electron transport in photosystem $\mathrm{II}^{10}$. Ground-water atrazine concentration in the United States range between $0.0002 \mathrm{mg} / \mathrm{L}-0.05 \mathrm{mg} / \mathrm{L}$ with peaks of $1 \mathrm{mg} / \mathrm{L}$ reported whenever it rains heavily immediately after field applications ${ }^{11,12}$. Recent monitoring data indicate that the value of $0.053 \mathrm{mg} / \mathrm{L}$ represents the $99^{\text {th }}$ percentile of atrazine concentrations in most ground waters of the United States, with a cut-off of $0.1 \mathrm{mg} / \mathrm{L}$ used to assess environmental relevance ${ }^{13}$. Atrazine exerts adverse physiological effects on aquatic insects including mosquito larvae at concentrations ranging between $0.02-5 \mathrm{mg} / \mathrm{L}^{12,14}$. The effects may include reduced hatching success, lower emergence success, longer emergence times for females, declining species richness, and distorted sex ratio with significant male bias ${ }^{12,14}$. However, exposure to atrazine has also been associated with significantly larger Aedes albopictus, a precursor for enhanced vectorial capacity ${ }^{14}$. Soil bacterial taxa, including Pseduomonas and Clavibacter express atz $A,-B$ and $-C$ genes that encode enzymes that metabolize atrazine and use the constituents as nitrogen source ${ }^{15}$. Thus atrazine contaminated environments are likely to favor proliferation of bacterial communities that hydrolyze atrazine for energy source ${ }^{11,16-18}$.

${ }^{1}$ Department of Entomology, University of Illinois at Urbana-Champaign, 505S. Goodwin Ave, Urbana, IL, 61801, USA. ${ }^{2}$ llinois Natural History Survey, University of Illinois at Urbana-Champaign, 1816 S. Oak St., Champaign, IL, 61820, USA. ${ }^{3}$ Crop Bioprotection Research Unit, Agricultural Research Service, U.S. Department of Agriculture, 1815 N. University St., Peoria, IL, 61604, USA. ${ }^{凶}$ e-mail: elijahjuma@gmail.com 
Malathion, a broad-spectrum organophosphate, is an inhibitor of acetylcholinesterase that causes acute neurotoxicity to susceptible invertebrates, including insects, at high doses. Malathion is commonly used in agricultural pest and public health vector control in the United States, although its use for public health vector control has declined significantly due to toxicity concerns and resistance development in some mosquito vector spe$\operatorname{cies}^{8,9,19,20}$. It is toxic to most aquatic life at concentrations as low as $0.006 \mathrm{mg} / \mathrm{L}^{21}$. Reported physiological and behavioral effects of malathion on mosquito life history traits include prolonged development times, reduced interspecific larval competition, toxicity to larvae, and increased vector competence in some species ${ }^{22}$. Malathion concentrations ranging from $0.001 \mathrm{mg} / \mathrm{L}$ up to $1 \mathrm{mg} / \mathrm{L}$ have been reported in the groundwaters of the United States $8,21-23$

Permethrin is a neurotoxin that acts by disrupting the functioning of the voltage-gated sodium channels in aquatic invertebrates, including mosquito larvae. In the United States, there has been a steady increase in the usage of pyrethroids in agricultural pest control and public health vector control with permethrin accounting for between $45-60 \%$ of all pyrethroids used ${ }^{24,25}$ As a result, it is frequently detected in up to $75 \%$ of soils and aquatic sediments, albeit at relatively low concentrations ( $\sim 10 \mathrm{ng} / \mathrm{g}$ dry weight). Permethrin has previously been associated with acute toxicity to several invertebrate indicators of environmental health, including Chironomus tentans, and Hyalella azteca ${ }^{24,26}$. Studies of its effects on mosquito aquatic stages are limited. However, in one study permethrin was associated with male-biased sex ratio in Aedes aegypti mosquitoes ${ }^{27}$. Permethrin concentrations ranging from $0.001-0.002 \mathrm{mg} / \mathrm{L}$ have been determined to be effective in routine mosquito control programs $\mathrm{s}^{27,28}$.

The role of pesticides used in agricultural pest control and public health vector control, in shaping the microbial community composition in the larval environment, and subsequently, the mosquito gut microbial colonization pattern, has received very little attention ${ }^{29}$. Previous studies have mostly investigated biotic endpoints, including microbial productivity, enzymatic activity, biomass, and respiration ${ }^{30-32}$. The lone study that applied next-generation sequencing to evaluate the effect of pesticides on microbial community composition focused on the larval environment, but not the mosquito gut bacterial communities ${ }^{29}$.

In this study, we used an experimental microcosm approach to evaluate whether environmentally relevant concentrations of atrazine, permethrin, and malathion in mosquito larval habitats play an important role in shaping the bacterial communities of mosquitoes and their larval environment. We used Culex pipiens L. and Aedes albopictus, two container-dwelling mosquitoes, as the model species for this study ${ }^{33-36}$. The two species thrive in diverse aquatic habitats embeded within agricultural landscapes, and thus are likely to be exposed to pesticides from agricultural applications or public health vector control initiatives ${ }^{37}$. Culex pipiens L. is an introduced European species that arrived in North America in the early $16^{\text {th }}$ Century through trade, and has been naturalized in the United States north of $39^{\circ}$ latitude ${ }^{38-40}$. It serves as both an amplifying and bridge vector for West Nile virus and St. Louis encephalitis due to its preference for feeding on birds ${ }^{41}$. Aedes albopictus is an invasive species first reported in the United States in August 1985 in Harris County, Texas, and has so far been reported in over 27 states $^{36,42,43}$. It is a known vector of dengue and Chikungunya viruses ${ }^{44}$, but experimental studies indicate that it can be a competent vector of up to 32 other viruses, 13 of which are present in the United States ${ }^{36}$.

We used Illumina MiSeq sequencing of the V3-V4 hypervariable regions of the bacterial 16S rRNA gene to determine how environmentally relevant concentrations of atrazine, malathion and permethrin affect the microbial communities of the two mosquito species and their larval environment. We hypothesized that: 1). Environmentally relevant concentrations of atrazine, permethrin and malathion will mediate a shift in the mosquito gut microbial community structure due to their toxic effect on the aquatic microbial communities; 2). Environmentally relevant concentrations of atrazine, permethrin and malathion will reduce mosquito gut microbial diversity by promoting the growth of microbial taxa that use pesticides as a source of carbon while eliminating pesticide-susceptible bacterial taxa. This study provides a basis for future studies investigating the responses of bacterial communities in the larval environment and the mosquito gut to exposures to chemical pesticides varied at different concentrations and the potential implications on mosquito ecology.

\section{Results}

Sequence processing and alpha diversity. Illumina sequencing of the V3-V4 hypervariable region of the 16S rRNA gene amplicons yielded a total of 7,147,541 raw sequence reads from 560 samples (200 Ae. albopictus; 200 Cx. pipiens L.; 160 water samples). After removing chimeric and other non-bacterial sequences, and quality filtering to remove bacterial OTUs constituting $<0.005 \%$ of the total sequences and rarefying to an even depth of 1002 sequences per sample to standardize sample size, a total of 466 samples (138 Ae. albopictus; 182 Cx. pipiens L.; 146 water samples) were retained. The final sample size consisted of a total of 7,064,485 sequences $($ mean \pm SE 15,733.82 $\pm 2933.51 ; \min =358 ; \max =944,292)$ that were clustered into 449 bacterial OTUs at $97 \%$ sequence similarity, which is adequate for bacterial identification to the genus level.

Microbial communities associated with Aedes albopictus. Rarefaction curve analysis (an interpolation and extrapolation method for comparing species richness based on samples of equal sizes) ${ }^{45,46}$ indicated that most OTUs were recovered by the sequencing depth coverage of 1002 sequences. Chaol estimator, an abundance-based diversity index for estimating rare species, revealed that up to $80.7 \% \pm 0.01$ (mean $\pm \mathrm{SE}$ ) of the OTUs were recovered. Bacterial OTU richness was highest in larval samples exposed to permethrin, and lowest in adult samples exposed to permethrin (Fig. S1). The observed OTUs, expected OTU richness (Chao1), and OTU diversity were significantly higher in larval samples compared to adult samples (Observed OTUs: Kruskal-Wallis chi-squared $=153.34, \mathrm{df}=15, p<0.0001$; Chao1: Kruskal-Wallis chi-squared $=156.15, \mathrm{df}=15, p<0.0001$; Shannon index: Kruskal-Wallis chi-squared $=172.14$, df $=15, p<0.0001$ ) (Fig. S2A).

Taxonomic classification and bacterial composition across pesticide treatments. To ascertain relative abundance of the bacterial taxa at each taxonomic level the 449 bacterial OTUs were classified into 


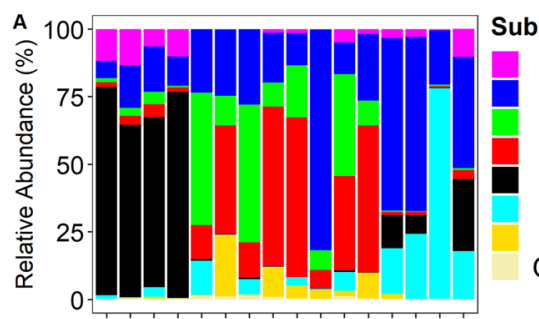

Subphylum

Actinobacteria

Alphaproteobacteria

Bacteroidetes

Betaproteobacteria

Firmicutes

Gammaproteobacteria

Verrucomicrobia

Other

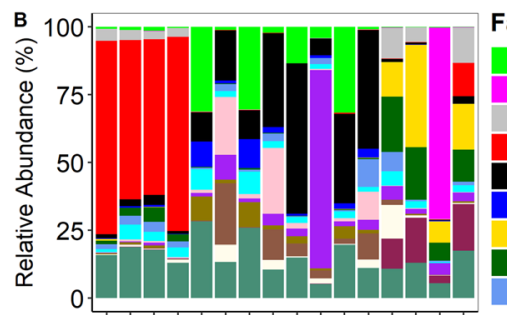

Family

[Weeksellaceae]

Aeromonadaceae

Bacillaceae

Clostridiaceae

Comamonadaceae

Flavobacteriaceae

Methylobacteriaceae

Rhizobiaceae

Rhizobiales

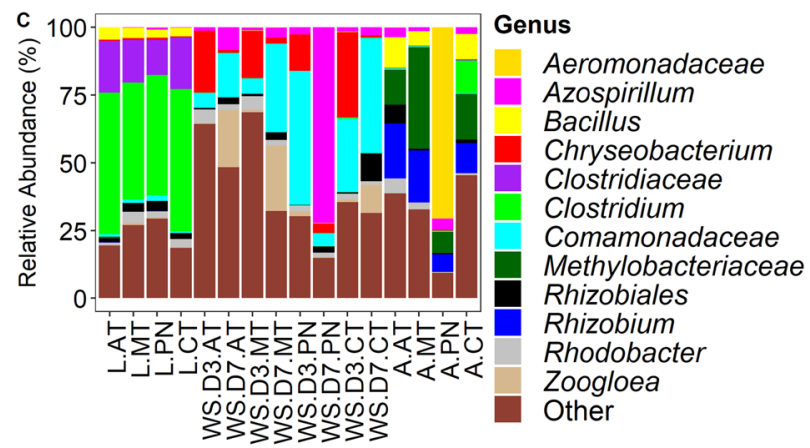

Rhodobacteraceae

Rhodocyclaceae

Rhodospirillaceae

Sphingomonadaceae

Verrucomicrobiaceae

Xanthobacteraceae

Xanthomonadaceae

Other

Figure 1. Composition of bacterial communities in Ae. albopictus and water samples. Taxa with sequence abundance $<1 \%$ of total sequences were pooled together as "Other" in all the taxonomic ranks. Panel A - relative abundance at subphylum level; panel B - relative abundance at family level; panel C - relative abundance at genus level. L - Larvae; A - Adults; AT - Atrazine; MT - Malathion; PN - Permethrin; CT Control; WS - Water Sample; D3 - Day 3; D7 - Day 7. Figures were generated using R version 3.6.1 ${ }^{76}$ (https:// www.r-project.org/) within the RStudio environment version 1.2.1335 $5^{77}$ (https://rstudio.com/).

seven phyla, 20 classes, 72 families, and 104 genera. The most dominant phyla were Proteobacteria (51.4\%), Firmicutes (28.3\%), and Bacteroidetes (12.1\%), and the rest were $<10 \%$, cumulatively (Fig. 1A). At the family level, Clostridiaceae (25.8\%) Comamonadaceae (13.9\%) and Rhodospirillaceae (10.3\%) were dominant while the rest were $<10 \%$, cumulatively (Fig. 1B). At the genus level, Clostridium (19.2\%), unclassified Comamonadaceae $(11.7 \%)$ and Azospirillum (10.0\%) were dominant while the rest were $<10 \%$, cumulatively (Fig. $1 \mathrm{C}$ ). Bacterial OTUs classified as Wolbachia were dominant overall at the genus level but were excluded from the analysis because Wolbachia is a maternally inherited obligate intracellular microbial symbiont naturally occurring in Ae. albopictus ${ }^{47}$, therefore it was not considered a gut bacteria for the purposes of the analyses. Overall, 183 (43.9\%) OTUs were shared among larvae, adults, and water samples from all pesticide treatments. Twenty-five, 14 , and 11 bacterial OTUs were unique to larval, adult, and water samples, respectively (Fig. S4A). Three hundred and seventy-one bacterial OTUs were detected in larval samples compared to 253 in adult samples, and 324 and 320 in day three water samples (WS.D3) and day seven water samples (WS.D7), respectively. The differences in OTUs detected per sample were statistically significant (Chi-squared $=22.3, \mathrm{df}=3, p<0.0001$ ). Multiple pairwise comparison with Bonferroni corrections revealed three statistically different sample groups (larvae vs. adults, $p<0.0001$; adult vs. WS.D7, $p=0.019$; adult vs. WS.D7, $p=0.031$ ).

Non-metric multidimensional scaling (NMDS) analysis using Bray-Curtis distance matrix revealed three clusters separating the microbial community composition by sample type (larvae, adult or water samples), and water sampling date (D3 vs. D7) but not by pesticide treatment (Fig. 3). These differences were confirmed by statistically significant pairwise comparisons using multi-response permutation procedure (MRPP) analysis with Bonferroni-adjusted p-values (Table 1). This is a non-parametric test for testing the hypothesis of no difference between two or more groups. Non-parametric multivariate analysis was performed to test for the effect of pesticide treatment and the day of water sampling on the water chemistry parameters. There were significant variations temperature, dissolved oxygen, conductivity, and total dissolved oxygen, for day 3 and 7 water samples but varied by pesticide treatment (Fig. S5, Table S1).

Microbial communities associated with Culex pipiens $L$. Rarefaction curve analysis recovered most of the OTUs by the sequencing depth coverage of 1002 sequences. Chaol estimator analysis detected up to $86.0 \%$ \pm 0.01 (mean \pm SE) of the expected bacterial OTUs. Bacterial OTU richness was highest in day three water 


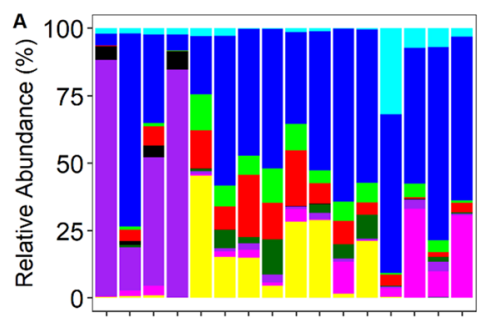

\author{
Subphylum \\ Actinobacteria \\ Alphaproteobacteria \\ Bacteroidetes \\ Betaproteobacteria \\ Cyanobacteria \\ Deltaproteobacteria \\ Firmicutes \\ Gammaproteobacteria \\ Verrucomicrobia \\ Other \\ Family
}

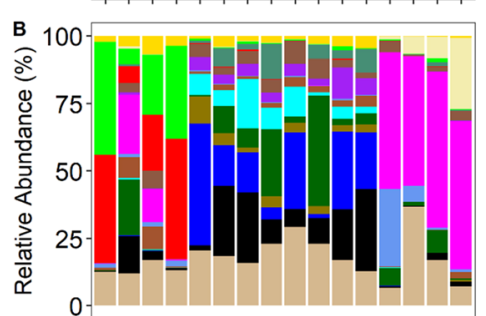

Acetobacteraceae

Aeromonadaceae

Bacillaceae

Bdellovibrionaceae

Clostridiaceae

Comamonadaceae

Hyphomicrobiaceae

Methylobacteriaceae

Microbacteriaceae

Rhodobacteraceae

Rhodocyclaceae

Rhodospirillaceae

Sphingobacteriaceae

Verrucomicrobiaceae

Xanthobacteraceae

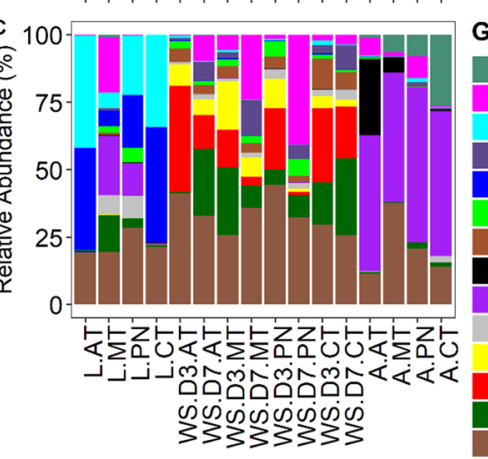

Genus
\begin{tabular}{|} 
Aeromonadaceae \\
Azospirillum \\
Bacillus \\
Bdellovibrio \\
Clostridium \\
Comamonadaceae \\
Devosia \\
Leucobacter \\
Methylobacteriaceae \\
Rhodobacter \\
Rhodocyclaceae \\
Verrucomicrobiaceae \\
Xanthobacter \\
Other
\end{tabular}

Figure 2. Composition of bacterial communities in Cx. pipiens L. and water samples. Taxa with sequence abundance $<1 \%$ of total sequences were pooled together as "Other" in all the taxonomic ranks. Panel A - relative abundance at subphylum level; panel B - relative abundance at family level; panel C - relative abundance at genus level. L - Larvae; A - Adults; AT - Atrazine; MT - Malathion; PN - Permethrin; CT Control; WS - Water Sample; D3 - Day 3; D7 - Day 7. Figures were generated using R version 3.6.1 ${ }^{76}$ (https:// www.r-project.org/) within the RStudio environment version 1.2.1335 $7^{77}$ (https://rstudio.com/).

\begin{tabular}{|l|l|l|l|l|l|l|l|l|l|l|l|l|}
\hline \multicolumn{4}{|l|}{ Sample type } & \multicolumn{3}{l}{ Ad } & \multicolumn{3}{l}{ N } & T & A & \multicolumn{2}{l|}{$\boldsymbol{P}$} \\
\hline L & vs. & A & 0.12 & vs. & 0.26 & 81 & vs. & 57 & -83.75 & 0.44 & $<0.001$ \\
\hline L & vs & WS.D3 & 0.12 & vs. & 0.17 & 81 & vs. & 36 & -67.25 & 0.38 & $<0.001$ \\
\hline L & vs & WS.D7 & 0.12 & vs. & 0.21 & 81 & vs. & 37 & -68.48 & 0.38 & $<0.001$ \\
\hline A & vs & WS.D3 & 0.26 & vs. & 0.17 & 57 & vs. & 36 & -56.54 & 0.46 & $<0.001$ \\
\hline A & vs & WS.D7 & 0.26 & vs. & 0.21 & 57 & vs. & 37 & -55.21 & 0.44 & $<0.001$ \\
\hline WS.D3 & vs & WS.D7 & 0.17 & vs. & 0.21 & 36 & vs. & 37 & -30.50 & 0.29 & $<0.001$ \\
\hline
\end{tabular}

Table 1. MRPP results showing differences in bacterial communities of Ae. albopictus mosquitoes by life stage and water sample type. Bacterial communities differed significantly between groups (larvae vs. adult samples vs. water samples). L - Larvae; A - Adults; WS - Water Sample; D3 - Day 3; D7 - Day 7. Ad average within-group distances for mosquito species $N$ sample size $T$-test statistic describing separation within groups. $A$ chancecorrected within-group agreement as $\log _{10}$.

samples exposed to permethrin, and lowest in adult samples exposed to malathion (Fig. S3). The observed OTUs, expected OTU richness (Chao1), and OTU diversity were significantly higher in larval samples compared to adult samples (Observed OTUs: Kruskal-Wallis chi-squared $=176.77, \mathrm{df}=15, p<0.0001$; Chaol: Kruskal-Wallis chi-squared $=186.53, \mathrm{df}=15, p<0.0001$; Shannon index: Kruskal-Wallis chi-squared $=165.47, \mathrm{df}=15$, $p<0.0001$ ) (Fig. S2B).

Taxonomic classification and bacterial composition across pesticide treatments. The most dominant phyla were Proteobacteria (60.5\%) and Firmicutes (25.7\%), the rest were $<10 \%$, cumulatively (Fig. 2A). At the family level Methylobacteriaceae (26.7\%), Clostridiaceae (11.85\%) and Bacillaceae (10.96\%), were dominant, the rest were $<10 \%$, cumulatively (Fig. 2B). At the genus level the unclassified Methylobacteriaceae (26.5\%), 
A

Stress $=0.16$
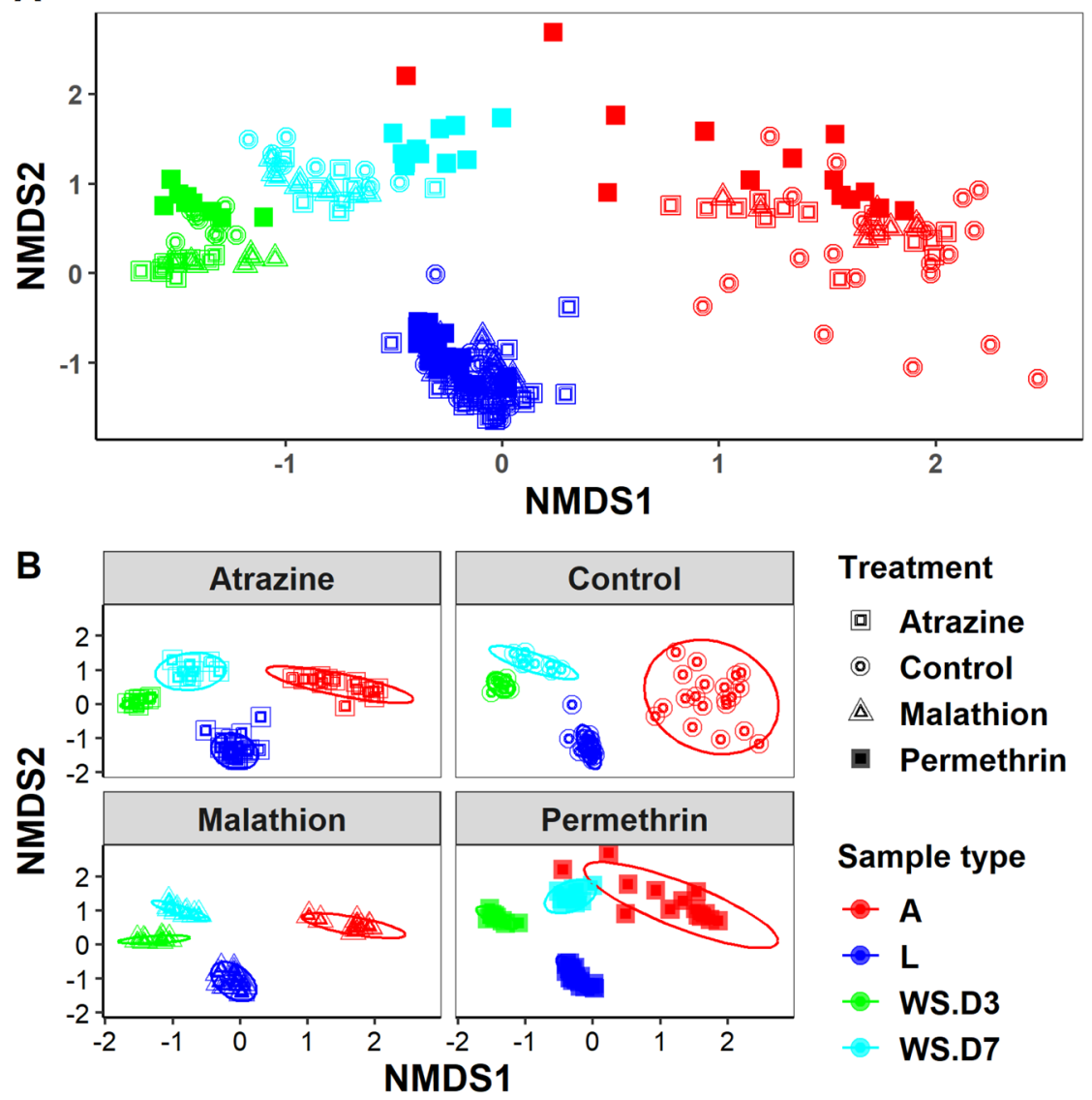

Treatment

(atrazine

() Control

$\triangle$ Malathion

- Permethrin

Sample type

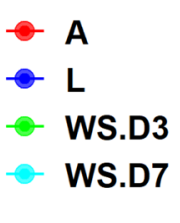

Figure 3. NMDS based on Bray-Curtis distance ordination of bacterial communities from Ae. albopictus larval, adult, and water samples from pesticide treatments and control group. (A) - all mosquito and water samples presented together; (B) - mosquito and water samples partitioned by the treatment or control from which they were obtained. L - Larvae; A - Adults; WS - water samples; D3 - Day 3; D7 - Day 7. Figures were generated using R version 3.6.1 ${ }^{76}$ (https://www.r-project.org/) within the RStudio environment version 1.2.1335 ${ }^{77}$ (https:// rstudio.com/).

Clostridium (11.03\%), Bacillus (10.58\%), were dominant while the rest were $<10 \%$, cumulatively (Fig. 2C). Overall, 258 (58.6\%) OTUs were shared among larvae, adults, and water samples from all pesticide treatments (Fig. S4B). Four-hundred and six bacterial OTUs were detected in larval samples compared to 394 in adult samples, and 329 and 335 in WS.D3 and WS.D7, respectively. The differences in OTUs detected per sample were statistically significant (Chi-squared $=12.9, \mathrm{df}=3, p<0.005$ ). Multiple pairwise comparison with Bonferroni corrections revealed only one statistically different sample group (larvae vs. WS.D3, $p=0.027$ ).

Non-metric multidimensional scaling (NMDS) analysis revealed three clusters separating the microbial community composition by sample type (larvae, adult or water samples), and water sampling date (D3 vs. D7) but not by pesticide treatment (Fig. 4). These differences were confirmed by statistically significant pairwise comparisons using MRPP analysis with Bonferroni-adjusted p-values (Table 2). There were significant variations in all the physical chemical parameters evaluated for day 3 and 7 water samples but varied by pesticide treatments (Fig. S6, Table S2).

\section{Discussion}

This study evaluated the effect of exposure to environmentally relevant concentrations of three pesticides, atrazine, permethrin, and malathion, on the diversity and composition of the gut bacterial communities of two container-dwelling mosquitoes, Ae. albopictus and Cx. pipiens L. Unexpectedly, malathion and permethrin treatments were associated with higher bacterial richness in larval mosquito samples and some water samples, for both Ae. albopictus and Cx. pipiens L. The lowest bacterial richness was reported in adult mosquito samples regardless of the pesticide treatment. Our study findings did not support our initial hypothesis that exposure to environmentally relevant pesticide concentrations could also induce a shift in the mosquito gut microbial community structure. However, there was evidence of distinct clustering of bacterial OTUs based on the mosquito life stage (larvae vs. adults) and the date in which water samples were collected from the microcosms (D3 vs. D7).

We report significantly higher bacterial richness associated with malathion and permethrin treatments in both larval samples and water samples, contrary to our initial hypothesis that exposure to environmentally 
A

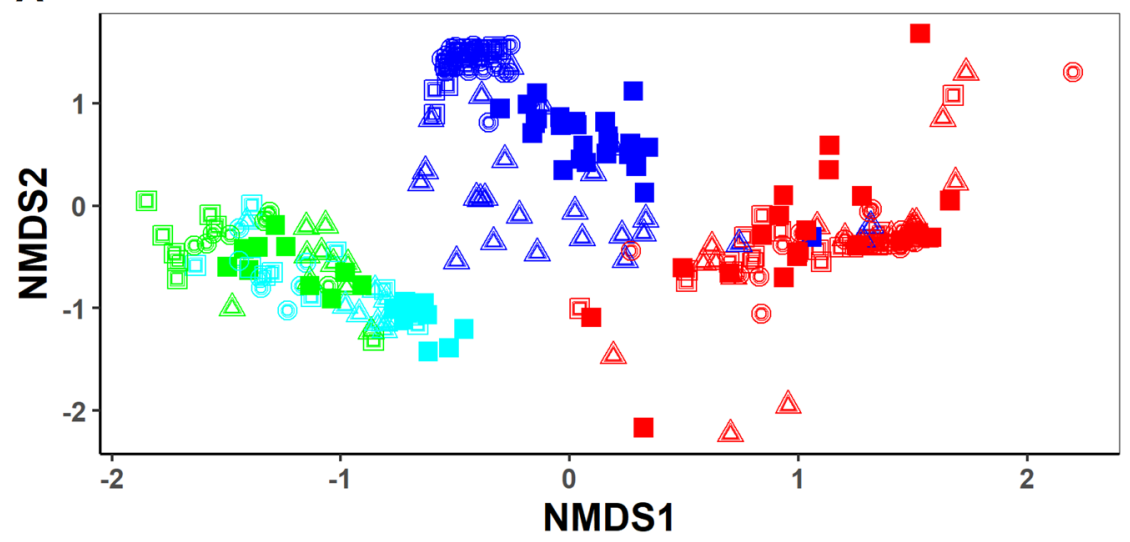

B
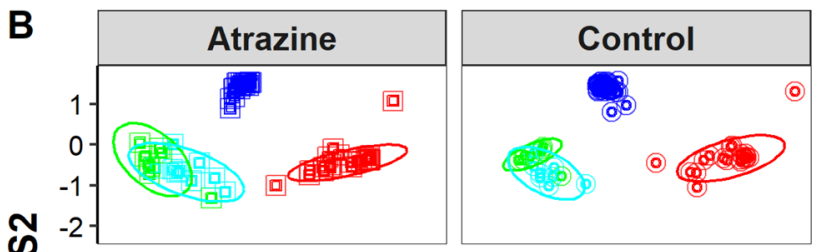

Treatment

(a) Atrazine

(6) Control

乏

-2
0
-1
-2

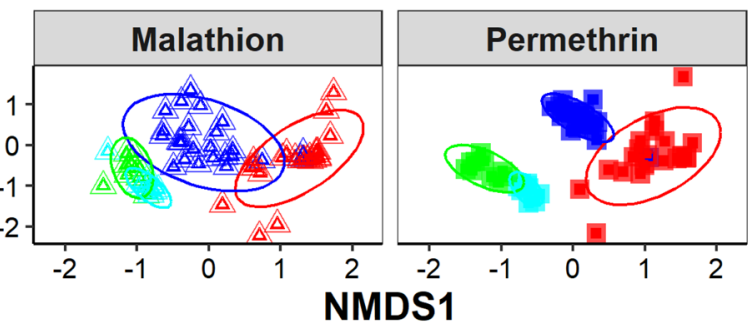

$\triangle$ Malathion

- Permethrin

\section{Sample type}

- A

- L

- WS.D3

WS.D7

Figure 4. NMDS based on Bray-Curtis distance ordination of bacterial communities from Cx. pipiens L. larval, adult, and water samples from pesticide treatments and control group. (A) - all mosquito and water samples presented together; (B) - mosquito and water samples partitioned by the treatment or control from which they were obtained. L - larvae; A - Adult; WS - water samples; D3 - Day 3; D7 - Day 7. Figures were generated using R version 3.6.1 $1^{76}$ (https://www.r-project.org/) within the RStudio environment version 1.2.1335 ${ }^{77}$ (https:// rstudio.com/).

\begin{tabular}{|c|c|c|c|c|c|c|c|c|c|c|c|}
\hline \multicolumn{3}{|c|}{ Sample type } & \multicolumn{3}{|l|}{ Ad } & \multicolumn{3}{|l|}{$\mathbf{N}$} & \multirow{2}{*}{$\begin{array}{l}\mathbf{T} \\
-11.60\end{array}$} & \multirow{2}{*}{\begin{tabular}{|l|} 
\\
0.12 \\
\end{tabular}} & \multirow{2}{*}{\begin{tabular}{|l|} 
\\
$<0.001$
\end{tabular}} \\
\hline WS.D3 & vs. & WS.D7 & 0.22 & $\begin{array}{l}\text { vs. } \\
\end{array}$ & $\mid \begin{array}{l}0.22 \\
\end{array}$ & 36 & \begin{tabular}{|l|} 
vs. \\
\end{tabular} & 37 & & & \\
\hline WS.D3 & vs. & $\mathrm{L}$ & 0.22 & vs. & 0.21 & 36 & vs. & 94 & -66.76 & 0.34 & $<0.001$ \\
\hline WS.D3 & vs. & A & 0.22 & vs. & 0.28 & 36 & vs. & 88 & -69.51 & 0.37 & $<0.001$ \\
\hline WS.D7 & vs. & $\mathrm{L}$ & 0.22 & vs. & 0.21 & 37 & vs. & 94 & -63.07 & 0.32 & $<0.001$ \\
\hline WS.D7 & vs. & A & 0.22 & vs. & 0.28 & 37 & vs. & 88 & -64.74 & 0.35 & $<0.001$ \\
\hline L & vs. & A & 0.21 & vs. & 0.28 & 94 & vs. & 88 & -85.64 & 0.33 & $<0.001$ \\
\hline
\end{tabular}

Table 2. MRPP results showing differences in bacterial communities of $C x$. pipiens $\mathrm{L}$. mosquitoes by life stage and water sample type. Bacterial communities differed significantly between groups (larvae vs. adult vs. water samples). L - Larvae; A - Adults; WS - Water Sample; D3 - Day 3; D7 - Day 7 Ad average within-group distances for mosquito species $N$ sample size $T$-test statistic describing separation within groups $A$ chancecorrected within-group agreement as $\log _{10}$.

relevant pesticide concentrations will reduce mosquito gut microbial diversity. Bacterial communities vary in their sensitivity to chemical pesticides ${ }^{48}$. Malathion in the natural environment has a short half-life ranging from a few hours to 1 week. It is broken down by several bacterial taxa into $\propto$ and $\beta$-malathion monoacid, malathion dicarboxylic acid, O,O-dimethyl phosphorodithioic acid, and diethyl maleate ${ }^{19,21}$. Permethrin is degraded to 3-phenoxybealdehyde and 3-phenoxybenzoic acid in the natural environment. Unlike malathion, permethrin is more stable in the environment than malathion and has the potential to exert its toxic effect over a longer period. However, the effect of permethrin may be diminished by its tendency to adsorb to dissolved solids or sediment surfaces and thus reduce the bioavailable concentrations able to induce bacterial toxicity ${ }^{24,49}$. We speculate that 
the increase in bacterial diversity in permethrin treatments may be the result of permethrin favoring enrichment of more bacterial species that degrade it or by eliminating dominant taxa that inhibit the growth of rare species communities ${ }^{30,32,50}$. Even though the concentrations of permethrin and malathion we used induced mortality in the mosquito larvae, it is likely that the fraction that was bioavailable to act on bacterial communities in the microcosms and in the mosquito guts was too low to induce significant shift in the bacterial richness and composition. Due to the low bioavailable amount, it is possible that the bacterial communities recovered rapidly from the initial short-term pulse of pesticide exposure.

Exposure of bacterial communities to pesticides in the environment produce variable effects that may be dependent on many factors, including the pesticide concentrations used, pesticide type, the physical and chemical characteristics of the study environment, and the study system (e.g., lake, estuary, or microcosm) ${ }^{29,31,50}$. Under certain conditions pesticides may promote bacterial diversity in the aquatic environments, partly due to the pesticides eliminating protozoa that graze on bacteria, thus releasing the bacterial communities from grazing pressure $^{48,51}$. We suspect that this phenomenon may have been at play in the permethrin and malathion treatments hence the higher bacterial diversity observed. Widenfal et al. (2008), using two different concentrations ("low" - $150 \mu \mathrm{g} / \mathrm{kg}$, and "higher" - 1000 times higher than the low concentrations), showed a lack of direct correlation between pesticide concentration and shifts in bacterial community composition. For instance, they reported shifts in bacterial community composition with captan and glyphosate at "low" concentrations but not "high" concentrations, possibly indicating that low concentrations possibly stimulate bacterial taxa that use pesticide as carbon source, or the low pesticide concentration eliminate susceptible taxa thus releasing the tolerant bacterial taxa from competition. When the experiment was monitored over a 30-day duration, there was clustering of bacterial communities between samples exposed to low vs. high concentrations from the different chemical pesticides tested. In this present study, only one pesticide concentration was applied per treatment, which may not be effective for monitoring shifts in bacterial community structure over time. Mosquitoes aquatic stages may be exposed to repeated short-term pulses of pesticides of varying concentrations during peak agricultural season or vector control initiatives. As such, additional studies with repeated pesticide exposures over longer periods will be helpful in decoupling bacterial communities' responses to pesticide exposure in the mosquito guts and the larval environment.

Some of the bacterial taxa regularly isolated in the mosquito gut, including Bacillus, Aeromonas, Flavobacterium, Xanthomonas sp. and Pseudomonas sp, have been implicated in the biodegradation of malathion and permethrin insecticides in the natural environment ${ }^{19,21,25,49,52,53}$. Our study isolated Flavobacterium, Pseudomonas and Bacillus water samples and the mosquito samples. In Ae. albopictus experiments, Bacillus occurred in small proportions in larval samples, while Flavobacterium was present in small fraction in water samples regardless of the pesticide treatment. Whereas these bacterial taxa have been associated with malathion and permethrin degradation, evidence from our study does not point to them exploiting this characteristic to bias their own proliferation at the expense of permethrin- or malathion-sensitive taxa. This further explains the lack of reported reduction in bacterial richness in the microcosm or in the mosquito gut microbiota that would be attributable to proliferation of some bacterial taxa over others due to the effect of the pesticides.

We observed shifts in the bacterial community composition between larval and adult mosquito samples. Similarly, significantly higher bacterial diversity was observed in larval stages compared to adult stages, in line with previous studies ${ }^{54-58}$. It is well established that mosquitoes acquire a substantial fraction of their microbial commensals from their environment as larvae, or through imbibing of the larval water at metamorpho$\operatorname{sis}^{59-63}$. During metamorphosis a portion of the mosquito microbial symbionts may be shed or sequestered due to modifications to the meconial peritrophic membrane; others may be lost through digestion and excretion, thus rendering them undetectable $e^{64-66}$. This provides a possible explanation to the observed shift in the microbial diversity and composition between the larval and adult mosquitoes. In this study, the adult mosquito samples were preserved immediately post-emergence. Thus, their microbial assemblages could only have been acquired transstadially, vertically, or by imbibing of the larval water at emergence ${ }^{64}$. The physiological states in the midguts of larvae, compared to adult mosquitoes, such as $\mathrm{pH}$, redox conditions, digestion, assimilation, and excretion rates, also potentially support the proliferation of distinct microbial assemblages ${ }^{60,67,68}$. This could provide an additional source of variation in the microbial composition and diversity between larvae and adult mosquitoes.

Microcosm studies provide an ideal starting point for understanding interactions between organisms and their environment since the experimental conditions can be carefully controlled. In the natural environment, factors such as nutrient pollutants from fertilizer residues can alter the aquatic microbial community response to pesticides by either stimulating or inhibiting the action of pesticides on the microbial communities ${ }^{48,69}$. Additionally, the interaction among a suite of pesticides in the natural environment is common and may also have similar stimulatory or inhibitory effects. Our experimental design evaluated each pesticide individually, yet sometimes significant disruptions of microbial communities may only be possible where a mixture of pesticides is involved as could be the case in the natural environment. Evidence from previous studies have also shown that factors such as adsorption of pesticides to dissolved solids in the aquatic environment affect the bioavailable amount that can induce shifts in aquatic or soil bacterial communities. Additionally, in the natural environment, pesticides undergo photolytic breakdown, resulting in intermediate compounds that affect the microorganisms differently compared to the parent compound ${ }^{15,30,48,70}$. However, we did not evaluate this in our study. The interpretation of our experiment is also limited by the fact that the experiment only characterized the microbial communities of the mosquito samples that survived the pesticide treatments. Additional future characterization of the microbial communities of the dead and surviving sample treatments is likely to be informative in helping to disentangle the selective effect of chemical pesticides on microbial community composition and diversity. Such studies should also be done across a broader range of pesticide concentrations to capture the full range of lethal and sublethal outcomes. 
In summary, we have shown that environmentally relevant concentrations of permethrin and malathion are associated with higher bacterial diversity in larval mosquito samples, but no detectable shift in the mosquito microbial community composition was reported for any of the pesticides evaluated. The effect of pesticides on the microbial community composition and diversity in the aquatic environments, and subsequently in the mosquito guts, can be complex and difficult to predict and may vary significantly depending on pesticide type, concentrations used and even the system being studied ${ }^{29,30,48}$. Similar studies in the future using both culture-independent and culture-dependent methods should vary pesticide concentrations and stagger the application to account for pesticide degradation and disappearance in the study system. This study forms a basis for future studies evaluating the effect of varied pesticide concentrations on the larval environment, and the mosquito gut bacterial communities and the potential implications on mosquito biology and ecology.

\section{Materials and Methods}

Mosquito sampling and colony establishment. Oviposition traps (ovitraps) were established in ten selected sites within residential neighborhoods in Champaign County, IL, for the sampling of Cx. pipiens and Ae. albopictus eggs. Three ovitraps were established at each of the ten selected sites for a total of 30 ovitraps. The traps consisted of 19L white cylindrical buckets with five $80 \mathrm{~mm}$-diameter overflow holes $18 \mathrm{~cm}$ from the base. Each ovitraps was baited with three liters of 3-day old grass infusion prepared following the method described in Jackson et al. ${ }^{71}$. To sample Ae. albopictus eggs, each ovitrap was lined with strips of germination paper and placed in shaded areas under trees or shrubs. Sampling was conducted twice every week between July 3 and September 20, 2017. The germination papers containing the sampled eggs were individually hatched in media consisting of $700 \mathrm{ml}$ of warm $\left(25 \pm 1^{\circ} \mathrm{C}\right)$ deionized water, $0.25 \mathrm{~g}$ of CM0001 Nutrient Broth (Oxoid, Hampshire, England) and $0.05 \mathrm{~g}$ yeast in enamel pans ${ }^{72}$. Emerging adult mosquitoes were identified to species using morphological characteristics and Ae. albopictus, the most abundant Aedes species in the samples, was selected for this experiment. The adults were pooled in a paperboard cage and artificially blood fed on citrated bovine blood. The resulting eggs were hatched and used for the microcosm experiment.

Culex pipiens egg rafts were sampled concurrently using the same ovitraps used for Ae. albopictus sampling. Egg rafts were collected using a paintbrush and placed on moist filter papers in a petri dish and transported to the laboratory. In the laboratory, egg rafts were hatched separately in Petri dishes and first instar larvae of $C x$. pipiens L. were distinguished from Cx. restuans (approx. 12-15 hours old) based on the presence of a clear scale anterior to the sclerotized egg-breaker in $C x$. restuans but not in $C x$. pipiens $\mathrm{L}^{39}$. First instar larvae of the identified Culex pipiens L. were pooled regardless of the ovitrap origin and used in the microcosm experiment.

Microcosm experiment. A completely randomized experimental design was used, consisting of four pesticide treatments (atrazine, malathion, permethrin, control). Two sets of experiments were established for each mosquito species. In the first set, first instar larvae were introduced to each microcosm and their development monitored up to late $4^{\text {th }}$ instar larvae after which a minimum of 15 larval samples surviving from each treatment were preserved at $-80^{\circ} \mathrm{C}$ for larval microbiome analysis. In the second set, first instar larvae were added to each microcosm and reared to adulthood. Newly emerging adults were preserved at $-80^{\circ} \mathrm{C}$ for adult microbiome analysis. Each treatment was replicated 5 times for a total of 80 containers. For each mosquito species, 50 first instar larvae were randomly assigned to each replicate. The microcosms consisted of $1 \mathrm{~L}$ of grass infusion in $5 \mathrm{~L}$ cylindrical containers. Each pesticide was diluted in acetone to make stock solutions. In the Cx. pipiens L. study, on day 0 , each microcosm was treated with the requisite concentration of the stock solution of each target pesticide diluted in $500 \mu \mathrm{L}$ acetone to make a final concentration of $5 \mathrm{mg} / \mathrm{L}$ atrazine, $0.05 \mathrm{mg} / \mathrm{L}$ malathion and $0.03 \mathrm{mg} / \mathrm{L}$ permethrin. Larval mortality was monitored over a 24 -hour period. Since no visible larval mortality was observed after the first 24 hours, an equal concentration of malathion and permethrin in $500 \mu \mathrm{L}$ of acetone were added to their respective treatments to make a final concentration of $0.1 \mathrm{mg} / \mathrm{L}$ malathion, and $0.06 \mathrm{mg} / \mathrm{L}$ permethrin, respectively. The average larval mortality by pesticide treatment in the microcosms ranged between 6-69.2\% (Fig. S7A). Similarly, in microcosm experiments for Ae. albopictus, after 24 hours when no mortality was observed, an equal concentration of malathion in $500 \mu \mathrm{L}$ of acetone was added to make a final concentration of $0.1 \mathrm{mg} / \mathrm{L}$ malathion. The average larval mortality by pesticide treatment in the microcosms ranged between $11.6-56.8 \%$ (Fig. S7B). For each control experiment, an equivalent volume of $500 \mu \mathrm{L}$ acetone was added to each control microcosm. The atrazine and malathion concentrations used in this experiment were based on concentrations previously reported from the environment ${ }^{16,73,74}$. The concentrations used for permethrin were determined by conducting preliminary bioassays with both $C x$. pipiens $\mathrm{L}$. and Ae. albopictus to determine $\mathrm{LC}_{50}$ values. The $\mathrm{LC}_{50}$ values obtained in our bioassays are similar to those from previous studies that have been found to induce acute toxicity in mosquito larval stages ${ }^{27,28}$.

Microcosms were maintained under controlled conditions of $27^{\circ} \mathrm{C} \pm 2$ and $\sim 75 \pm 5 \%$ relative humidity. In the adult mosquito experimental component, emergence data were recorded, and at least ten newly emerged females were collected per replicate and preserved at $-80^{\circ} \mathrm{C}$ for later microbial DNA analysis. In addition, aliquots of $30 \mathrm{~mL}$ grass infusion samples were collected from all microcosms on days three and seven and preserved at $-80^{\circ} \mathrm{C}$ to compare the microbiota of the mosquito guts to the aquatic environment in the microcosm. Physiochemical parameters of the microcosms, including $\mathrm{pH}$, temperature, conductivity, salinity, total dissolved solids, and dissolved oxygen, were taken using ExStik meters (Extech Instruments, Nashua, NH).

DNA extraction, 16 S rRNA gene library preparation and sequencing. Frozen mosquito samples were surface-sterilized in $70 \%$ ethanol solution for 5 minutes, transferred to $3 \%$ bleach solution for 3 minutes, and then once more in $70 \%$ ethanol for another 5 minutes. Samples were then rinsed five times in sterile water and once in $0.8 \%$ saline solution to rid them of any alcohol ${ }^{56}$. Dissections were carried out under a stereomicroscope. Each gut sample was dissected in a drop of physiological saline solution on a sterile microscope slide 
and used for DNA extraction. Fifty mosquito samples were used per treatment: 25 larval samples and 25 adult samples divided evenly among each treatment replicate. Water samples were thawed, and the total volume of $30 \mathrm{~mL}$ centrifuged at $5000 \mathrm{rpm}$ for $20 \mathrm{mins}$ at $3^{\circ} \mathrm{C}$ (Centrifuge $5810 \mathrm{R}$, Eppendorf, Hamburg, Germany), and microbial DNA extracted from each sample pellet. Midgut samples and water samples were individually suspended in bead solution of PowerSoil DNA Isolation Kit, homogenized using Retsch MM 300 TissueLyser (Retsch, Haan, Germany) and genomic DNA extracted using DNeasy PowerLyzer PowerSoil DNA Isolation Kit (Qiagen Inc., Valencia, CA) according to the manufacturer's instructions. DNA was quantified using Nanodrop 1000 (ThermoFisher Scientific, Pittsburgh, PA). The V3-V4 hypervariable region of the bacterial 16S rRNA gene was PCR-amplified using the following primer set specific for V3-V4 region of the 16S rRNA gene: Forward 5'CCTACGGGNGGCWGCAG; Reverse 5'GACTACHVGGGTATCTAATCC. PCR was done in $25 \mu \mathrm{L}$ reactions containing $12.5 \mu \mathrm{L}$ of $2 \mathrm{x}$ KAPA HiFi HotStart ReadyMix, $5 \mu \mathrm{L}$ of $1 \mu \mathrm{M}$ each of the forward and reverse primers, and $2.5 \mu \mathrm{L}$ of template genomic DNA. PCR conditions were $95^{\circ} \mathrm{C}$ for $3 \mathrm{~min} ; 25$ cycles of: $95^{\circ} \mathrm{C}$ for $30 \mathrm{~s}, 55^{\circ} \mathrm{C}$ for $30 \mathrm{~s}, 72^{\circ} \mathrm{C}$ for $30 \mathrm{~s} ; 72^{\circ} \mathrm{C}$ for 5 mins; hold at $4^{\circ} \mathrm{C}$. PCR amplicons were cleaned using AMPure XP beads to remove free primers and primer-dimer species, and a second PCR was conducted using Nextera XT Index Kit (Illumina, San Diego, CA) to attach dual indices and Illumina sequencing adapters ${ }^{72}$. Index PCR was conducted in $45 \mu \mathrm{L}$ reactions containing $25 \mu \mathrm{L}$ of $2 x$ KAPA HiFi HotStart ReadyMix, $5 \mu \mathrm{L}$ each of index 1 and index 2 combinations, and $10 \mu \mathrm{L}$ of PCR grade water. Thermocycling conditions were $95^{\circ} \mathrm{C}$ for $3 \mathrm{~min} ; 8$ cycles of $95^{\circ} \mathrm{C}$ for $30 \mathrm{~s}, 55^{\circ} \mathrm{C}$ for $30 \mathrm{~s}, 72^{\circ} \mathrm{C}$ for $30 \mathrm{~s} ; 72^{\circ} \mathrm{C}$ for $5 \mathrm{mins}$; hold at $4{ }^{\circ} \mathrm{C}$. A negative control sample made up of DNA extracted from molecular biology grade water was sequenced with the same protocol to allow detection of the contamination background. PCR amplicons were cleaned and normalized using a SequalPrep normalization plate (Thermofisher Scientific, Waltham, MA). The pooled library was mixed with Phix control spike-in of $5 \%$ as a sequencing control. The samples were sequenced using an Illumina MiSeq system with a MiSeq V3 $2 \times 300$ bp sequencing kit. The demultiplexed reads were quality-trimmed to Q30 using CLC genomics workbench v8.5 (Qiagen Inc., Valencia, CA). Read pairing, fixed-length trimming and OTU clustering were done using CLC Bio Microbial Genomics module (Qiagen Inc., Valencia, CA) using the reference sequences from the Greengenes ribosomal RNA gene database $\mathrm{e}^{75}$.

Statistical analysis. All analyses were conducted using R version 3.6.1 ${ }^{76}$ within the RStudio environment version 1.2.1335 77 , and PC-ORD version $6.08^{78}$ statistical packages. Bacterial OTUs consisting of $<0.005 \%$ of the total sequences were removed prior to analysis to eliminate artifacts of PCR and sequencing ${ }^{79}$. Sequences were rarefied to a depth of 1002 reads per sample to correct for unequal sample sizes. From a starting sample size of 560 samples, 94 samples had $<1002$ reads and were removed from further analysis. Rarefaction curves were fitted to estimate sample coverage ${ }^{80,81}$ using the "phyloseq" package version 1.28.0 in $\mathrm{R}^{82}$. Rarefaction curves were generated using unrarefied data at a maximum read depth of 1002 sequences. Alpha diversity metrics, including Shannon diversity index, observed species, and chaol estimator were generated in QIIME $2^{83}$, and their means and $95 \%$ confidence intervals calculated to test for significant differences in alpha diversity indices between pesticide treatments. Kruskal-Wallis test was used to test for differences in the means between sample types and pairwise Wilcoxon rank-sum test with Bonferroni correction performed to separate significant pesticide treatments. Beta-diversity measures were computed using the Bray-Curtis dissimilarity index using the "phyloseq" package and non-metric multidimensional scaling (NMDS) ordination plots generated to visualize the results. A multi-response permutation procedure (MRPP) was conducted in PC-ORD version $6.08^{78}$ to determine significant differences in microbial communities between pesticide treatments. The p-values were Bonferroni-corrected to reduce the chances of type I error. Non-parametric multivariate analysis was performed to test for the effect of pesticide treatment and the day of water sampling on the water chemistry parameters, using the function "nonpartest" from the R package "npmv" version 2.4.0. Significant treatment factors across water chemistry parameters were delineated using the function "ssnonpartest" $"$. Venn diagrams were constructed using the R package "limma" ${ }^{85}$ version 3.40.2 to visualize OTUs that were shared between larvae, adults and water samples for both Cx. pipiens L. and Ae. albopictus samples.

Received: 3 December 2019; Accepted: 11 May 2020;

Published online: 11 June 2020

\section{References}

1. Hazen, T. C., Rocha, A. M., Techtmann, S. M., Steffan, R. J. \& Ramos, J. L. Advances in monitoring environmental microbes. Curr. Opin. Biotechnol. 24, 526-533 (2013).

2. Gotelli, N. \& Colwell, R. Estimating species richness. In Biological Diversity. Frontiers in Measurement and Assessment (ed. Magurran and B. J. McGill) 39-54 https://doi.org/10.2307/3547060 (Oxford University Press, 2011).

3. Lozupone, C. A., Hamady, M., Kelley, S. T. \& Knight, R. Quantitative and qualitative $\beta$ diversity measures lead to different insights into factors that structure microbial communities. Appl. Environ. Microbiol. 73, 1576-1585 (2007).

4. Toccalino, P. L., Gilliom, R. J., Lindsey, B. D. \& Rupert, M. G. Pesticides in groundwater of the United States: decadal-scale changes, 1993-2011. Ground Water 52, 112-125 (2014).

5. Nkya, T. E., Akhouayri, I., Kisinza, W. \& David, J.-P. Impact of environment on mosquito response to pyrethroid insecticides: Facts, evidences and prospects. Insect Biochem. Mol. Biol. 43, 407-416 (2013).

6. Harbison, J. E. et al. Standardized operational evaluations of catch basin larvicides from seven mosquito control programs in the midwestern United States during 2017. J. Am. Mosq. Control Assoc. 34, 107-116 (2018).

7. Imfeld, G. \& Vuilleumier, S. Measuring the effects of pesticides on bacterial communities in soil: A critical review. Eur. J. Soil Biol. 49, 22-30 (2012).

8. Bonner, M. R. et al. Malathion exposure and the incidence of cancer in the agricultural health study. Am. J. Epidemiol. 166, 1023-1034 (2007).

9. Stark, P. M., Fredregill, C. L., Nolan, M. S. \& Debboun, M. Field cage insecticide resistance tests against Culex quinquefasciatus Say (Diptera: Culicidae) in Harris County, Texas, USA. J. Vector Ecol. 42, 279-288 (2017). 
10. Gruessner, B. \& Watzin, M. C. Response of aquatic communities from a Vermont stream to environmentally realistic atrazine exposure in laboratory microcosms. Environ. Toxicol. Chem. 15, 410-419 (1996).

11. Downing, H. F. et al. Effects of the agricultural pesticides atrazine, chlorothalonil, and endosulfan on South Florida microbial assemblages. Ecotoxicology 13, 245-260 (2004).

12. Graymore, M., Stagnitti, F. \& Allinson, G. Impacts of atrazine in aquatic ecosystems. Environ. Int. 26, 483-495 (2001)

13. Van Der Kraak, G. J., Hosmer, A. J., Hanson, M. L., Kloas, W. \& Solomon, K. R. Effects of atrazine in fish, amphibians, and reptiles: An analysis based on quantitative weight of evidence. Critical Reviews in Toxicology 44, 1-66 (2014).

14. Bara, J. J., Montgomery, A. \& Muturi, E. J. Sublethal effects of atrazine and glyphosate on life history traits of Aedes aegypti and Aedes albopictus (Diptera: Culicidae). Parasitol. Res. 113, 2879-2886 (2014).

15. De Souza, M. L. et al. Molecular basis of a bacterial consortium: Interspecies catabolism of atrazine. Appl. Environ. Microbiol. 64, 178-184 (1998).

16. Muturi, E. J., Orindi, B. O. \& Kim, C. H. Effect of leaf type and pesticide exposure on abundance of bacterial taxa in mosquito larval habitats. PLoS One 8, e71812 (2013).

17. Jason Krutz, L. et al. Agronomic and environmental implications of enhanced s-triazine degradation. Pest Manag. Sci. 66, 461-481 (2010).

18. Delorenzo, M. E., Lauth, J., Pennington, P. L., Scott, G. I. \& Ross, P. E. Atrazine effects on the microbial food web in tidal creek mesocosms. Aquat. Toxicol. 46, 241-251 (1999).

19. Alvarenga, N., Birolli, W. G. \& Porto, A. L. M. Biodegradation of organophosphate and pyrethroid pesticides by microorganims. In Pollutants in Buildings, Water and Living Organisms vol. 7 85-121 (Springer International Publishing Switzerland, 2015).

20. Dye-braumuller, K., Fredregill, C. \& Debboun, M. Mosquito control. in Mosquitoes, Communities, and Public Health in Texas (eds. Debboun, M., Nava, M. R. \& Rueda, L.) 249-278 https://doi.org/10.1016/B978-0-12-814545-6.00008-0 (Elsevier Inc., 2020).

21. Singh, B., Kaur, J. \& Singh, K. Microbial degradation of an organophosphate pesticide, malathion. Crit Rev Microbiol 40, 1040-841 (2014).

22. Muturi, E. J., Costanzo, K., Kesavaraju, B. \& Alto, B. W. Can pesticides and larval competition alter susceptibility of Aedes mosquitoes (Diptera: Culicidae) to arbovirus infection? J. Med. Entomol. 48, 429-436 (2011).

23. Muturi, E. J., Costanzo, K., Kesavaraju, B., Lampman, R. \& Alto, B. W. Interaction of a pesticide and larval competition on life history traits of Culex pipiens. Acta Trop. 116, 141-146 (2010).

24. Amweg, E. L., Weston, D. P. \& Ureda, N. M. Use and toxicity of pyrethroid pesticides in the central valley, California, USA. Environ. Toxicol. Chem. 24, 966-972 (2005).

25. Thatheyus, A. J. \& Gnana Selvam, A. D. Synthetic pyrethroids: Toxicity and biodegradation. Appl. Ecol. Environ. Sci. 1, 33-36 (2013).

26. Ding, Y., Harwood, A. D., Foslund, H. M. \& Lydy, M. J. Distribution and toxicity of sediment-associated pesticides in urban and agricultural waterways from Illinois, USA. Environ. Toxicol. Chem. 29, 149-157 (2010).

27. Mebrahtu, Y. B., Norem, J. \& Taylor, M. Inheritance of larval resistance to permethrin in Aedes aegypti and association with sex ratio distortion and life history variation. Am. J. Trop. Med. Hyg. 56, 456-465 (1997).

28. Milam, C. D., Farris, J. L. \& Wilhide, J. D. Evaluating mosquito control pesticides for effect on target and nontarget organisms. Arch. Environ. Contam. Toxicol. 39, 324-328 (2000).

29. Muturi, E. J., Donthu, R. K., Fields, C. J., Moise, I. K. \& Kim, C.-H. Effect of pesticides on microbial communities in container aquatic habitats. Sci. Rep. 7, 44565 (2017).

30. Widenfalk, A., Bertilsson, S., Sundh, I. \& Goedkoop, W. Effects of pesticides on community composition and activity of sediment microbes - responses at various levels of microbial community organization. Environ. Pollut. 152, 576-584 (2008).

31. Widenfalk, A., Svensson, J. M. \& Goedkoop, W. Effects of the pesticides captan, deltamethrin, isoproturon, and primicarb on the microbial community of a freshwater sediment. Env Tox Chem 23, 1920-1927 (2004).

32. Päiviö, J. Effects of modern pesticides on the microbial community in a natural and in an artificial sediment-a microcosm study. Rapport-Sveriges Lantbruksuniversitet, Institutionen ... (Swedish University of Agricultural Sciences, 1999).

33. Helbing, C. M., Moorhead, D. L., Mitchell, A. L. \& Mitchell, L. Population dynamics of Culex restuans and Culex pipiens (Diptera: Culicidae) related to climatic factors in northwest Ohio. Environ. Entomol 44, 1022-1028 (2015).

34. Lampman, R. L. \& Novak, R. J. Oviposition preferences of Culex pipiens and Culex restuans for infusion-baited traps. J. Am. Mosq. Control Assoc. 12, 23-32 (1996).

35. Reiter, P. A standardized procedure for the quantitative surveillance of certain Culex mosquitoes by egg raft collection. J. Am. Mosq. Control Assoc. 2, 219-21 (1986)

36. Vanlandingham, D. L., Higgs, S. \& Huang, Y.-J. S. Aedes albopictus (Diptera: Culicidae) and mosquito-borne viruses in the United States. J. Med. Entomol. 53, 1024-1028 (2016).

37. Lenormand, T., Bourguet, D., Guillemaud, T. \& Raymond, M. Tracking the evolution of insecticide resistance in the mosquito Culex pipiens. Nature 400, 861-864 (1999).

38. Joyce, A. L., Melese, E., Ha, P. T. \& Inman, A. Population genetic structure of the Culex pipiens (Diptera: Culicidae) complex, vectors of West Nile virus, in five habitats. Parasites and Vectors 11 (2018).

39. Reiskind, M. H. \& Wilson, M. L. Interspecific competition between larval Culex restuans Theobald and Culex pipiens L. (Diptera: Culicidae) in Michigan. J. Med. Entomol. 45, 20-27 (2008).

40. Ross, H. H. The colonization of temperate North America by mosquitoes and man. Mosq. News 24, 103-118 (1964).

41. Kunkel, K. E., Novak, R. J., Lampman, R. L. \& Gu, W. Modeling the impact of variable climatic factors on the crossover of Culex restauns and Culex pipiens (Diptera: Culicidae), vectors of West Nile virus in Illinois. Am. J. Trop. Med. Hyg. 74, 168-173 (2006).

42. Carolina, S. et al. Overwintering and establishment of Aedes albopictus (Diptera: Culicidae) in an urban La Crosse virus enzootic site in Illinois. J. Med. Entomol. 37, 559-570 (2000).

43. Moore, C. G. \& Mitchell, C. J. Aedes albopictus in the United States: Ten-year presence and public health implications. Emerg. Infect. Dis. 3, 329-334 (1997).

44. Johnson, T. L. et al. Modeling the Environmental Suitability for Aedes (Stegomyia) aegypti and Aedes (Stegomyia) albopictus (Diptera: Culicidae) in the contiguous United States. J. Med. Entomol. 54, 1605-1614 (2017).

45. Chao, A. et al. Rarefaction and extrapolation with Hill numbers: A framework for sampling and estimation in species diversity studies. Ecol. Monogr. 84, 45-67 (2014).

46. Crist, T. O. \& Veech, J. A. Additive partitioning of rarefaction curves and species-area relationships: Unifying a-, - and??-diversity with sample size and habitat area. Ecol. Lett. 9, 923-932 (2006).

47. Chouin-Carneiro, T. et al. Wolbachia strain $w$ AlbA blocks Zika virus transmission in Aedes aegypti. Med. Vet. Entomol. 34, 116-119 (2020).

48. DeLorenzo, M. E., Scott, G. I. \& Ross, P. E. Toxicity of pesticides to aquatic microorganisms: A review. Environ. Toxicol. Chem. 20, $84-98(2001)$

49. Zhan, H., Huang, Y., Lin, Z., Bhatt, P. \& Chen, S. New insights into the microbial degradation and catalytic mechanism of synthetic pyrethroids. Environ. Res. 182, 109138 (2020).

50. Staley, Z. R., Harwood, V. J. \& Rohr, J. R. A synthesis of the effects of pesticides on microbial persistence in aquatic ecosystems. Critical Reviews in Toxicology 45, 813-836 (2015).

51. Johnsen, K., Jacobsen, C., Torsvik, V. \& Sørensen, J. Pesticide effects on bacterial diversity in agricultural soils - a review. Biol. Fertil. Soils 33, 443-453 (2001). 
52. Lee, S., Gan, J., Kim, J. S., Kabashima, J. N. \& Crowley, D. E. Microbial transformation of pyrethroid insecticides in aqueous and sediment phases. Environ. Toxicol. Chem. 23, 1-6 (2004).

53. Bhatt, P., Huang, Y., Zhan, H. \& Chen, S. Insight into microbial applications for the biodegradation of pyrethroid insecticides. Front. Microbiol. 10, 1-19 (2019).

54. Gimonneau, G. et al. Composition of Anopheles coluzzii and Anopheles gambiae microbiota from larval to adult stages. Infect. Genet. Evol. 28, 715-724 (2014).

55. Wang, Y., Gilbreath, T. M., Kukutla, P., Yan, G. \& Xu, J. Dynamic gut microbiome across life history of the malaria mosquito Anopheles gambiae in Kenya. PLoS One 6, 1-9 (2011).

56. Coon, K. L., Vogel, K. J., Brown, M. R. \& Strand, M. R. Mosquitoes rely on their gut microbiota for development. Mol. Ecol. 23, 2727-2739 (2014).

57. Dickson, L. B. et al. Carryover effects of larval exposure to different environmental bacteria drive adult trait variation in a mosquito vector. Sci. Adv. 3 (2017).

58. Coon, K. L., Brown, M. R. \& Strand, M. R. Mosquitoes host communities of bacteria that are essential for development but vary greatly between local habitats. Mol. Ecol. 25, 5806-5826 (2016).

59. David, M. R., Santos, L. M. B., Dos, Vicente, A. C. P. \& Maciel-de-Freitas, R. Effects of environment, dietary regime and ageing on the dengue vector microbiota: evidence of a core microbiota throughout Aedes aegypti lifespan. Mem. Inst. Oswaldo Cruz 111, $577-587$ (2016)

60. Briones, A. M., Shililu, J., Githure, J., Novak, R. \& Raskin, L. Thorsellia anophelis is the dominant bacterium in a Kenyan population of adult Anopheles gambiae mosquitoes. ISME J. 2, 74-82 (2008).

61. Zouache, K. et al. Bacterial diversity of field-caught mosquitoes, Aedes albopictus and Aedes aegypti, from different geographic regions of Madagascar. FEMS Microbiol. Ecol. 75, 377-389 (2011)

62. Osei-Poku, J., Mbogo, C. M., Palmer, W. J. \& Jiggins, F. M. Deep sequencing reveals extensive variation in the gut microbiota of wild mosquitoes from Kenya. Mol. Ecol. 21, 5138-5150 (2012).

63. Duguma, D. et al. Bacterial communities associated with Culex mosquito larvae and two emergent aquatic plants of bioremediation importance. PLoS One 8, 1-11 (2013).

64. Lindh, J. M., Borg-Karlson, A. K. \& Faye, I. Transstadial and horizontal transfer of bacteria within a colony of Anopheles gambiae (Diptera: Culicidae) and oviposition response to bacteria-containing water. Acta Trop. 107, 242-250 (2008).

65. Moll, R. M., Romoser, W. S., Modrzakowski, M. C., Moncayo, A. C. \& Lerdthusnee, K. Meconial peritrophic membranes and the fate of midgut bacteria during mosquito (Diptera: Culicidae) metamorphosis. J. Med. Entomol 38, 29-32 (2001).

66. Minard, G., Mavingui, P. \& Moro, C. Diversity and function of bacterial microbiota in the mosquito holobiont. Parasit. Vectors 6, 146 (2013).

67. Pang, X. et al. Mosquito C-type lectins maintain gut microbiome homeostasis. Nat. Microbiol. 1 (2016).

68. Andrews, E. S., Crain, P. R., Fu, Y., Howe, D. K. \& Dobson, S. L. Reactive oxygen species production and Brugia pahangi survivorship in Aedes polynesiensis with artificial Wolbachia infection types. PLoS Pathog. 8, 1003075 (2012).

69. Lozano, R. B. \& Pratt, J. R. Interaction of toxicants and communities: The role of nutrients. Environ. Toxicol. Chem. 13, 361-368 (1994).

70. Staley, Z. R., Senkbeil, J. K., Rohr, J. R. \& Harwood, V. J. Lack of direct effects of agrochemicals on zoonotic pathogens and fecal indicator bacteria. Appl. Environ. Microbiol. 78, 8146-8150 (2012).

71. Jackson, B. T., Paulson, S. L., Youngman, R. R., Scheffel, S. L. \& Hawkins, B. Oviposition preferences of Culex restuans and Culex pipiens (Diptera: Culicidae) for selected infusions in oviposition traps and gravid traps. J Am Mosq Control Assoc 21, 360-365 (2005).

72. Zheng, M. L., Zhang, D. J., Damiens, D. D., Lees, R. S. \& Gilles, J. R. L. Standard operating procedures for standardized mass rearing of the dengue and chikungunya vectors Aedes aegypti and Aedes albopictus (Diptera: Culicidae) - II - Egg storage and hatching. Parasites and Vectors 8, 348 (2015).

73. Vogel, A., Jocque, H., Sirot, L. K. \& Fiumera, A. C. Effects of atrazine exposure on male reproductive performance in Drosophila melanogaster. J Insect Physiol 72, 14-21 (2015).

74. Poupardin, R. et al. Cross-induction of detoxification genes by environmental xenobiotics and insecticides in the mosquito Aedes aegypti: Impact on larval tolerance to chemical insecticides. Insect Biochem. Mol. Biol. 38, 540-551 (2008).

75. DeSantis, T. Z. et al. Greengenes, a chimera-checked $16 \mathrm{~S}$ rRNA gene database and workbench compatible with ARB. Appl. Environ. Microbiol. 72, 5069-72 (2006).

76. R core team. R: a language and environment for statistical computing. R Foundation for Statistical Computing, Vienna, Austria. (2017) doi:ISBN 3-900051-07-0.

77. Rstudio Team. RStudio: Integrated development for R. (2016).

78. McCune, B. \& Mefford, M. J. PC-ORD Multivariate Analysis of Ecological Data. Version 6 User's Booklet https://doi. org/10.1890/0012-9623(2005)86[6a:MAOEDU]2.0.CO;2 (MjM Software, 2011).

79. Bokulich, N. A. et al. Quality-filtering vastly improves diversity estimates from Illumina amplicon sequencing. Nat. Methods 10, $57-59(2013)$.

80. Hughes, J. B., Hellmann, J. J., Ricketts, T. H. \& Bohannan, B. J. Counting the uncountable: statistical approaches to estimating microbial diversity. Appl. Environ. Microbiol. 67, 4399-406 (2001).

81. Olszewski, T. D. A unified mathematical framework for the measurement of richness and evenness within and among multiple communities. Oikos 104, 377-387 (2004).

82. McMurdie, P. J. \& Holmes, S. Phyloseq: an R package for reproducible interactive analysis and graphics of microbiome census data. PLoS One 8, e61217 (2013).

83. Caporaso, J. G. et al. QIIME allows analysis of high-throughput community sequencing data. Nat. Methods 7, 335-336 (2010)

84. Burchett, W. W., Ellis, A. R., Harrar, S. W. \& Bathke, A. C. Nonparametric inference for multivariate data: the R package npmv. J. Stat. Softw. 76 (2017).

85. Ritchie, M. E. et al. limma powers differential expression analyses for RNA-sequencing and microarray studies. Nucleic Acids Res. 43, e47-e47 (2015)

\section{Acknowledgements}

We thank Millon Blackshear for his technical assistance and all members of the Medical Entomology Laboratory (INHS), David Buendia, Yani Kaldis, Rosemary Ogbonna, and Eddie Lewis, for assisting with fieldwork and running the experiments, and members of Allan Laboratory for providing valuable reviews to the manuscript. This research was supported by the U.S. Department of Agriculture, Agricultural Research Service. Any opinions, findings, conclusions, or recommendations expressed in this publication are those of the author(s) and do not necessarily reflect the view of the U.S. Department of Agriculture. Mention of trade names or commercial products in this publication is solely for the purpose of providing specific information and does not imply recommendation or endorsement by the U.S. Department of Agriculture. USDA is an equal opportunity provider and employer. This work was supported by NSF DEB 1754115; Waste Tire Fund and Emergency Public Health Act from the State of Illinois; University of Illinois Institute for Sustainability, Energy, and Environment grant. 


\section{Author contributions}

E.O.J., E.J.M., B.F.A. conceived the study, and organized the study, E.O.J., conducted the experiment, analyzed the data, and wrote the manuscript, C.D. processed the sequencing data and generated the OTU table, E.J.M., and B.F.A., assisted with data analysis and interpretation. C.H.K. assisted with conducting the experiments, C.S., and E.J.M., contributed, reagents, materials, analysis tools. All authors read and approved the manuscript.

\section{Competing interests}

The authors declare no competing interests.

\section{Additional information}

Supplementary information is available for this paper at https://doi.org/10.1038/s41598-020-66452-5.

Correspondence and requests for materials should be addressed to E.O.J.

Reprints and permissions information is available at www.nature.com/reprints.

Publisher's note Springer Nature remains neutral with regard to jurisdictional claims in published maps and institutional affiliations.

(c) Open Access This article is licensed under a Creative Commons Attribution 4.0 International

License, which permits use, sharing, adaptation, distribution and reproduction in any medium or format, as long as you give appropriate credit to the original author(s) and the source, provide a link to the Creative Commons license, and indicate if changes were made. The images or other third party material in this article are included in the article's Creative Commons license, unless indicated otherwise in a credit line to the material. If material is not included in the article's Creative Commons license and your intended use is not permitted by statutory regulation or exceeds the permitted use, you will need to obtain permission directly from the copyright holder. To view a copy of this license, visit http://creativecommons.org/licenses/by/4.0/.

(c) The Author(s) 2020 